Cela posé, on montre que l'intégrale définie

$$
Z(x)=\int_{x_{0}}^{x} G(x, s) f(s) d s
$$

\section{Sur la détermination d'un système fondamental de solutions d'une équation différentielle linéaire d'ordre $n$}

\author{
par S. VASILACHE (București)
}

1. Considérons une équation différentielle linéaire d'ordre $n$, (1) $y^{(n)}(x)=a_{1}(x) y^{(n-1)}(x)+a_{2}(x) y^{(n-2)}(x)+\ldots+a_{n}(x) y(x)+f(x)$, où $\left(a_{i}(x)\right)_{1 \leqslant i \leqslant n}$ et $f(x)$ sont des fonctions numériques définies dans un intervalle fermé $I=[a, b]$ de la droite réelle $R$, ou des fonctions holomorphes dans un domaine connexe $U$ du plan complexe $C$, limité par une courbe $\Gamma$.

La solution générale de l'équation (1) est de la forme

$$
y(x)=\sum_{i=1}^{n} c_{i} Y_{i}(x)+Z(x),
$$

où $\left(c_{i}\right)_{1 \leqslant i \leqslant n}$ sont $n$ constantes arbitraires, $\left(Y_{i}\right)_{1 \leqslant i \leqslant n}$ est un système fondamental de solutions de l'équation homogène associée

$$
y^{(n)}(x)=a_{1}(x) y^{(n-1)}(x)+a_{2}(x) y^{(n-2)}(x)+\ldots+a_{n}(x) y(x)
$$

et $Z(x)$ une solution particulière de l'équation non homogène (1). Pour avoir la solution générale de (1) il faut donc, au préalable, déterminer un système fondamental de (3), ainsi qu'une solution particulière de (1). En ce qui concerne la solution particulière de (1), on sait (voir E. Goursat [1], II, p. 421-422) que si l'on connaît un système fondamental de solutions $\left(\bar{Y}_{i}\right)_{1 \leqslant i \leqslant n}$ de l'équation homogène associée (3), on peut en déduire une solution particulière $Z(x)$ de l'équation (1) par la méthode de Cauchy, en procédant comme suit:

On détermine les constantes $\left(c_{i}\right)_{1<i \leqslant n}$ de telle sorte que l'intégrale $\sum_{i=1}^{n} c_{i} Y_{i}(x)$ soit nulle, ainsi que ses $n-2$ premières dérivées, pour une valeur $s$ de $x$, tandis que la dérivée $(n-1)^{\text {ème }}$ se réduise à l'unité. L'intégrale ainsi obtenue, que nous désignons par $G(x, s)$, dépend naturellement de $x$ et de la valeur initiale $s$ et satisfait aux $n$ conditions

$$
G_{x i}^{(i)}(s, s)=0 \quad(i=0,1, \ldots, n-2), \quad G_{x n-1}^{(n-1)}(s, s)=1,
$$

où l'on fait $x=s$ après dérivation. prise depuis une limite fixe arbitraire $x_{0} \epsilon[a, b]$, est une solution particulière de l'équation (1), qui est nulle ainsi que ses $(n-1)$ premières dérivées pour $x=x_{0}$.

Dans le présent travail nous exposons une méthode de détermination d'un système fondamental, en montrant que la solution particulière $G(x, s)$ de l'équation homogène (3), qui satisfait aux $n$ conditions (4), de Cauchy donne non seulement la solution particulière (5), mais aussi un système fondamental de solutions de l'équation homogène associée (3). En d'autres mots, la solution particulière $G(x, s)$ permet d'obtenir la solution générale de l'équation (1).

Notre méthode est la suivante: On prend pour la fonction inconnue $y(x)$ le développement taylorien

$$
y(x)=\sum_{i=1}^{n} c_{i} \frac{\left(x-x_{0}\right)^{i-1}}{(i-1) !}+\int_{x_{0}}^{x} \frac{(x-s)^{n-1}}{(n-1) !} y^{(n-1)}(s) d s,
$$

où $\left(c_{i}\right)_{1 \leqslant i \leqslant n}$ sont $n$ constantes arbitraires, ce qui permet de transformer l'équation différentielle (1) en une équation intégrale du type Volterra de seconde espèce dont l'inconnue est la dérivée $y^{(n)}(x)$.

En désignant par $P(x, s)$ le noyau résolvant de cette équation intégrale et en posant

$$
G(x, s)=\frac{(x-s)^{n-1}}{(n-1) !}+\int_{x_{0}}^{x} \frac{\left(x-s_{1}\right)^{n-1}}{(n-1) !} P\left(s_{1}, s\right) d s_{1}
$$

on trouve que toute solution de l'équation (1) est de la forme

$y(x)=\sum_{i=1}^{n} c_{i}\left\{\frac{\left(x-x_{0}\right)^{i-1}}{(i-1) !}+\int_{x_{0}}^{x} G(x, s) L_{s} \frac{\left(s-x_{0}\right)^{i-1}}{(i-1) !} d s\right\}+\int_{x_{0}}^{x} G(x, s) f(s) d s$,

où $\left(c_{i}\right)_{1 \leqslant i \leqslant n}$ sont des constantes arbitraires.

On montre ensuite que la fonction $G(x, s)$ est la solution de l'équation différentielle homogène

$$
G_{x^{n}}^{(n)}(x, s)=a_{1}(x) G_{x^{n-1}}^{(n-1)}(x, s)+a_{2}(x) G_{x^{n-2}}^{(n-2)}(x, s)+\ldots+a_{n}(x) G(x, s)
$$

qui vérifie les conditions de Cauchy

$$
G_{x^{i}}^{(i)}(s, s)=0 \quad(i=0,1, \ldots, n-z), \quad G_{x^{n-1}}^{(n-1)}(s, s)=1,
$$

où l'on fait $x=s$ après dérivation. 
Compte tenu des propriétés de la fonction $G(x, s)$ on établit enfin que les fonctions

$$
Y_{i}(x)=\frac{\left(x-x_{0}\right)^{i-1}}{(i-1) !}+\int_{x_{0}}^{x} G(x, s) L_{s} \frac{\left(s-x_{0}\right)^{i-1}}{(i-1) !} d s \quad(i=1,2, \ldots, n)
$$

où

$$
\begin{aligned}
L_{s} \frac{\left(s-x_{0}\right)^{i-1}}{(i-1) !}= & \hat{a}_{1}(x) \frac{d^{n-1}}{d x^{n-1}}\left(\frac{\left(s-x_{0}\right)^{i-1}}{(i-1) !}\right)+ \\
& +a_{2}(x) \frac{d^{n-2}}{d x^{n-2}}\left(\frac{\left(s-x_{0}\right)^{i-1}}{(i-1) !}\right)+\ldots+a_{n}(x) \frac{\left(s-x_{0}\right)^{i-1}}{(i-1) !}
\end{aligned}
$$

forment un système fondamental de solutions de l'équation (3), et que

$$
Z(x)=\int_{x_{0}}^{x} G(x, s) f(s) d s
$$

est une solution particulière de l'équation non homogène (1).

\section{Transformation de l'équation (1) en une équation intégrale et} définition de la fonction $G(x, s)$. Désignons par $L y$ l'opérateur linéaire

$$
L y=a_{1}(x) y^{(n-1)}(x)+a_{2}(x) y^{(n-2)}(x)+\ldots+a_{n}(x) y(x)
$$

et écrivons l'équation (1) sous la forme

$$
y^{(n)}=L y+f(x)
$$

En prenant pour $y(x)$ le développement taylorien avec le reste sous la forme de Lagrange,

$$
y(x)=\sum_{i=1}^{n} c_{i} \frac{\left(x-x_{0}\right)^{i-1}}{(i-1) !}+\int_{x_{0}}^{x} \frac{(x-s)^{n-1}}{(n-1) !} y^{(n)}(s) d s,
$$

où $\left(c_{i}\right)_{1 \leqslant i \leqslant n}$ sont $n$ constantes arbitraires, l'équation (7) se transforme en une équation intégrale du type Volterra de seconde espèce dont l'inconnue est la dérivée $y^{(n)}(x)$.

En faisant tous les calculs, et en désignant respectivement par

les opératéurs:

$$
L_{x} \frac{(x-s)^{n-1}}{(n-1) !} \quad \text { et } \quad L_{x} \frac{\left(x-x_{0}\right)^{i-1}}{(i-1) !}
$$

(9) $\quad L_{x} \frac{(x-s)^{n-1}}{(n-1) !}=a_{1}(x) \frac{d^{n-1}}{d x^{n-1}}\left(\frac{(x-s)^{n-1}}{(n-1) !}\right)+$

$$
+a_{2}(x) \frac{d^{n-2}}{d x^{n-2}}\left(\frac{(x-s)^{n-1}}{(n-1) !}\right)+\ldots+a_{n}(x) \frac{(x-s)^{n-1}}{(n-1) !},
$$

(10) $L_{x} \frac{\left(x-x_{0}\right)^{i-1}}{(i-1) !}=a_{1}(x) \frac{d^{n-1}}{d x^{n-1}}\left(\frac{\left(x-x_{0}\right)^{i-1}}{(i-1) !}\right)+$

$$
\begin{array}{r}
+a_{2}(x) \frac{d^{n-2}}{d x^{n-2}}\left(\frac{\left(x-x_{0}\right)^{i-1}}{(i-1) !}\right)+\ldots+a_{n}(x) \frac{\left(x-x_{0}\right)^{i-1}}{(i-1) !} \\
=a_{n-i+1}(x)+a_{n-i+2}(x)\left(x-x_{0}\right)+\ldots+a_{n}(x) \frac{\left(x-x_{0}\right)^{i-1}}{(i-1) !} . \\
(i=1,2, \ldots, n)
\end{array}
$$

on trouve pour $y^{(n)}(x)$ l'équation intégrale

$$
\text { (11) } y^{(n)}(x)=\sum_{i=1}^{n} c_{i} L_{x} \frac{\left(x-x_{0}\right)^{i-1}}{(i-1) !}+\int_{x_{0}}^{x} L_{x} \frac{(x-s)^{n-1}}{(n-1) !} y^{(n)}(s) d s+f(x) \text {. }
$$

Si $P(x, s)$ est le noyau résolvant formé avec les itérés du noyau $L_{x}\left[(x-s)^{n-1} /(n-1) !\right]$ la solution de (11) est donnée par l'expression

$$
\begin{aligned}
y^{(n)}(x)= & f(x)+\sum_{i=1}^{n} c_{i} L_{x} \frac{\left(x-x_{0}\right)^{i-1}}{(i-1) !}+ \\
& +\int_{x_{0}}^{x} P(x, s)\left[f(s)+\sum_{i=1}^{n} c_{i} L_{x} \frac{\left(s-x_{0}\right)^{i-1}}{(i-1) !}\right] d s .
\end{aligned}
$$

D'autre part, en remplaçant dans le second membre de (8) la dérivée $y^{(n)}(s)$ par sai valeur (12) on trouve finalement pour la solution $y(x)$ de l'équation (1) l'expression

$$
\begin{array}{r}
y(x)=\sum_{i=1}^{n} c_{i} \frac{\left(x-x_{0}\right)^{i-1}}{(i-1) !}+\int_{x_{0}}^{x} \frac{(x-s)^{n-1}}{(n-1) !}\left\{f(s)+\sum_{i=1}^{n} c_{i} L_{x} \frac{\left(s-x_{0}\right)^{i-1}}{(i-1) !}+\right. \\
\left.\quad+\int_{x_{0}}^{s} P\left(s, s_{1}\right)\left[f(s)+\sum_{i=1}^{n} c_{i} L_{s_{1}} \frac{\left(s_{1}-x_{0}\right)^{i-1}}{(i-1) !}\right] d s_{1}\right\} d s,
\end{array}
$$

que l'on peut encore écrire sous la forme

$$
\text { (14) } \begin{aligned}
y(x)= & \sum_{i=1}^{n} c_{i}\left\{\frac{\left(x-x_{0}\right)^{i-1}}{(i-1) !}+\int_{x_{0}}^{x} \frac{(x-s)^{n-1}}{(n-1) !}\left[L_{s} \frac{\left(s-x_{0}\right)^{i-1}}{(i-1) !}+\right.\right. \\
& \left.\left.\quad+\int_{x_{0}}^{s} P\left(s, s_{1}\right) L_{s_{1}} \frac{\left(s_{1}-x_{0}\right)^{i-1}}{(i-1) !} d s_{1}\right] d s\right\}+ \\
& +\int_{x_{0}}^{x} \frac{(x-s)^{n-1}}{(n-1) !}\left[f(s)+\int_{x_{0}}^{s} P\left(s, s_{1}\right) f\left(s_{1}\right) d s_{1}\right] d s .
\end{aligned}
$$


Si l'on applique la formule de Dirichlet aux intégrales doubles du second membre de (14) il vient

$$
\begin{aligned}
& \int_{x_{0}}^{x} \frac{(x-s)^{n-1}}{(n-1) !} d s \int_{x_{0}}^{s} P\left(s, s_{1}\right) L_{s_{1}} \frac{\left(s_{1}-x_{0}\right)^{i-1}}{(i-1) !} d s_{1} \\
& =\int_{x_{0}}^{x} L_{s_{1}} \frac{\left(s_{1}-x_{0}\right)^{i-1}}{(i-1) !} d s_{1} \int_{s_{1}}^{x} \frac{(x-s)^{n-1}}{(n-1) !} P\left(s, s_{1}\right) d s \\
& \int_{x_{0}}^{x} \frac{(x-s)^{n-1}}{(n-1) !} d s \int_{x_{0}}^{s} P\left(s, s_{1}\right) f\left(s_{1}\right) d s_{1}=\int_{x_{0}}^{x} f\left(s_{1}\right) d s_{1} \int_{s_{1}}^{x} \frac{(x-s)^{n-1}}{(n-1) !} P\left(s, s_{1}\right) d s,
\end{aligned}
$$

et

de sorte que (14) s'écrit encore

(15) $y(x)=\sum_{i=1}^{n} c_{i}\left\{\frac{\left(x-x_{0}\right)^{i-1}}{(i-1) !}+\int_{x_{0}}^{x} L_{s} \frac{\left(s-x_{0}\right)^{i-1}}{(i-1) !}\left[\frac{(x-s)^{n-1}}{(n-1) !}+\right.\right.$

$$
\begin{array}{r}
\left.\left.+\int_{s}^{x} \frac{\left(x-s_{1}\right)^{n-1}}{(n-1) !} P\left(s_{1}, s\right) d s_{1}\right] d s\right\}+ \\
+\int_{x_{0}}^{x} f(s)\left[\frac{\left(x-s^{n-1}\right)}{(n-1) !}+\int_{s}^{x} \frac{\left(x-s_{1}\right)^{n-1}}{(n-1) !} P\left(s_{1}, s\right) d s_{1}\right] d s .
\end{array}
$$

En posant

$$
G(x, s)=\frac{(x-s)^{n-1}}{(n-1) !}+\int_{s}^{x} \frac{\left(x-s_{1}\right)^{n-1}}{(n-1) !} P\left(s_{1}, s\right) d s_{1},
$$

la solution $y(x)$ donnée par (15) prend la forme plus condensée

(17) $y(x)=\sum_{i=1}^{n} c_{i}\left\{\frac{\left(x-x_{0}\right)^{i-1}}{(i-1) !}+\int_{x_{0}}^{x} G(x, s) L_{s} \frac{\left(s-x_{0}\right)^{i-1}}{(i-1) !} d s\right\}+$

$$
+\int_{x_{0}}^{x} G(x, s) f(s) d s .
$$

Montrons maintenant que $\int_{x_{0}}^{x} G(x, s) f(s) d s$ est une solution particulière de l'équation non homogène (1), et que les fonctions qui multiplient les constantes $\left(e_{i}\right)_{1 \leqslant i \leqslant n}$ dans la somme du second membre de $(17)$ forment un système fondamental de solutions de l'équation homogène (3).

Pour cela nous allons d'abord étudier la fonction $G(x, s)$ définie par la relation (16).
3. Étude de la fonction $G(x, s)$. De l'expression (16) de $G(x, s)$ on tire, en dérivant par rapport à $\infty$

$$
\begin{aligned}
& G_{x^{i}}^{(i)}(x, s)=\frac{(x-s)^{n-i-1}}{(n-i-1) !}+\int_{s}^{x} \frac{\left(x-s_{1}\right)^{n-i-1}}{(n-i-1) !} P\left(s_{1}, s\right) d s \quad(i=0,1, \ldots, n-1), \\
& G_{x^{n-1}}^{(n-1)}(x, s)=1+\int_{s}^{x} P\left(s_{1}, s\right) d s_{1} \\
& G_{x^{n}}^{(n)}(x, s)=P(x, s) .
\end{aligned}
$$

et

Montrons maintenant que l'on a

$$
P(x, s)=L_{x} G(x, s) .
$$

En effet, le noyau $P(x, s)$ vérifie l'équation fonctionnelle.

$$
P(x, s)=L_{x} \frac{(x-s)^{n-1}}{(n-1) !}+\int_{s}^{x} L_{x} \frac{\left(x-s_{1}\right)^{n-1}}{(n-1) !} P\left(s_{1}, s\right) d s_{1} .
$$

D'autre part, la relation (16) donne, en lui appliquant l'opérateur $L_{x}$

$$
L_{x} G(x, s)=\int_{s}^{x} L_{x} \frac{(x-s)^{n-1}}{(n-1) !} P\left(s_{1}, s\right) d s_{1}+L_{x} \frac{(x-s)^{n-1}}{(n-1) !}
$$

d'où, compte tenu de $(21), L_{x} G(x, s)=P(x, s)$. Én remplaçant dans le second membre de l'équation (19), $P(x, s)$ par son expression (20), on trouve que le noyau $G(x, s)$ est solution de l'équation différentielle homogène (23) $G_{x^{n}}^{(n)}(x, s)=a_{1}(x) G_{x^{n-1}}^{(n-1)}(x, s)+a_{2}(x) G_{x^{n-2}}^{(n-2)}(x, s)+\ldots+a_{n}(x) G(x, s)$.

De plus, les relations (18) donnent, en $\mathrm{y}$ faisant $x=s$ après dérivation

$$
G_{x^{i}}^{(i)}(s, s)=0 \quad(i=0,1, \ldots, n-2), \quad G_{x^{n-1}}^{(n-1)}(s, s)=1 .
$$

Donc $G(x, s)$ est la solution de l'équation (23) qui vérifie les conditions de Cauchy (24).

Nous pouvons ainsi énoncer le

THÉoRÈme 1. Étant donnée l'équation différentielle (1) où $\left(a_{i}(x)\right)_{1 \leqslant i \leqslant n}$ et $f(x)$ sont des fonctions continues (resp. holomorphes) dans l'intervalle fermé $I=[a, b]$ de la. droite réellé $R$ (resp. dans un domaine connexe $U d u$ plan complexe $C$, limité par une courbe $\Gamma$ ), toute solution de (1) est de la forme (17) où $\left(c_{i}\right)_{1 \leqslant i \leqslant n}$ sont des constantes arbitraires et $G(x, s)$ est donné par. (16). Cette fonotion $G(x, s)$ est la solution de l'équation homogène (23) qui vérifie pour $x=s \epsilon[a, b]$ les conditions de Cauchy (24) où l'on fait $x=s$ après dérivation.

Annales Polonici Mathematici IIr. 
4. Détermination d'un système fondamental de solutions de l'équation homogène (3). Revenons à l'expression (17) de $y(x)$. En posant

$$
\begin{gathered}
Y_{i}(x)=\frac{\left(x-x_{0}\right)^{i-1}}{(i-1) !}+\int_{x_{0}}^{x} G(x, s) L_{s} \frac{\left(s-x_{0}\right)^{i-1}}{(i-1) !} d s \quad(i=1,2, \ldots, n), \\
Z(x)=\int_{x_{0}}^{x} G(x, s) f(s) d s,
\end{gathered}
$$

nous allons montrer que

a. $\left(Y_{i \leqslant i \leqslant n}\right.$ forment un système fondamental de solutions de l'équation homogène (3)

b. $Z(x)$ est une solution particulière de l'équation non homogène (1).

En effet, en calculant les dérivées des fonctions $\left(Y_{i}\right)_{i \leqslant i \leqslant n}$ jusqu'à celle d'ordre $n-1$, on trouve

$$
\begin{aligned}
& Y_{i}^{(k)}(x)=\frac{\left(x-x_{0}\right)^{i-k-1}}{(i-k-1) !}+\int_{x_{0}}^{x} G_{x^{k}}^{(k)}(x, s) L_{s} \frac{\left(s-x_{0}\right)^{i-1}}{(i-1) !} d s \\
& Y_{i}^{(k)}(x)=\int_{x_{0}}^{x} G_{x^{k}}^{(k)}(x, s) L_{s} \frac{\left(s-x_{0}\right)^{i-1}}{(i-1) !} d s \quad \text { pour } \quad 0 \leqslant k \leqslant i-1, \\
& Y_{i}^{(n)}(x)=L_{x} \frac{\left(x-x_{0}\right)^{i-1}}{(i-1) !}+\int_{x_{0}}^{x} G_{x^{n}}^{(n)}(x, s) L_{s} \frac{\left(s-x_{0}\right)^{i-1}}{(i-1) !} d s .
\end{aligned}
$$

D'autre part, l'opérateur $Y_{i}^{(n)}(x)-L Y_{i}(x)$ a pour expression, compte tenu des relations $(27)$ et $(24)$,

$$
\begin{aligned}
Y_{i}^{(n)}(x)-L_{x} Y_{i}(x)= & L_{x} \frac{\left(x-x_{0}\right)^{i-1}}{(i-1) !}+\int_{x_{0}}^{x} G_{x^{n}}^{(n)}(x, s) L_{s} \frac{\left(s-x_{0}\right)^{i-1}}{(i-1) !} d s- \\
& -L_{x} \frac{\left(x-x_{0}\right)^{i-1}}{(i-1) !}-\int_{x_{0}}^{x} L_{x} G(x, s) L_{s} \frac{\left(s-x_{0}\right)^{i-1}}{(i-1) !} d s=0
\end{aligned}
$$

car, d'après (23), on a $G_{x^{n}}^{(n)}-L_{x} G=0$.

Les fonctions $\left(Y_{i}(x)\right)_{1 \leqslant i \leqslant n}$ données par (25) sont donc des solutions particulières de l'équation homogène $y^{(n)}(x)=L y(x)$.

Montrons enfin que le système d'intégrales (25) est un système fondamental de solutions. Pour cela, il suffit de montrer (voir Goursat [1], I, p. 411) que le déterminant formé par les valeurs initiales de ces $n$ intégrales et de leurs $(n-1)$ premières dérivées pour un point non singulier $x_{0} \in[a, b]$ est différent de zéro.
Désignons par $\Delta$ ce déterminant, done

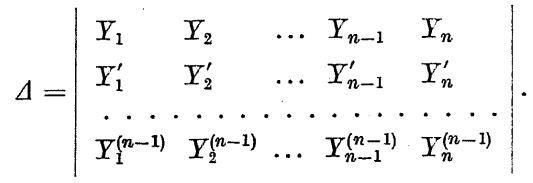

Compte tenu des relations (25) et $(27)$, on a

où

$$
\Delta=\left|m_{i k}\right| \text {, }
$$

$$
m_{j k}=\left\{\begin{array}{l}
\frac{\left(x-x_{0}\right)^{j-k}}{(j-k) !}+\int_{x_{0}}^{x} G_{x k-1}^{(k-1)} L_{0} \frac{\left(s-x_{0}\right)^{k-1}}{(k-1) !} d s \quad(k \geqslant j), \\
\int_{x_{0}}^{x} G_{x^{n-1}}^{(j-1)} L_{s} \frac{\left(s-s_{0}\right)^{k-1}}{(k-1) !} d s \quad(k<j) .
\end{array}\right.
$$

D'où l'on voit que pour $x=x_{0}$ on a $\Delta=1$. Par conséquent, le système de solutions (25) est un système fondamental.

Vérifions d'autre part que la fonction $Z(x)$ donnée par (26) est une solution particulière de l'équation (1) (cf. Goursat [1], p. 422). En effet, on a

$$
L_{x} Z(x)=\int_{x_{0}}^{x} L_{x} G(x, s) f(s) d s \quad \text { et } \quad Z^{(n)}(x)=f(x)+\int_{x_{0}}^{x} G_{x^{n}}^{(n)}(x, s) f(s) d s .
$$

d'où

$$
Z^{(n)}(x)-L_{x} Z(x)=f(x)+\int_{x_{0}}^{x}\left(G_{x^{n}}^{(n)}(x, s)-L_{x} G(x, s)\right) f(s) d s
$$

ou

$$
Z^{(n)}(x)-L_{x} Z(x)=f(x),
$$

Pour $x=x_{0}$, on a aussi (cf. (27)) $Y_{i}^{(k-1)}\left(x_{0}\right)=\delta_{i k}\left(\delta_{i k}\right.$ étant le symbole de Kronecker) et

$$
Z^{(k)}\left(x_{0}\right)=0 \quad(k=0,1, \ldots, n-1) .
$$

$\Pi$ en résulte que la somme du second membre de (17) est une solution de l'équation homogène (3). Nous avons donc démontré le

THÉoRÈme 2. Soit (1) une équation différentielle d'ordre $n$, où $\left(a_{i}(x)\right)_{1 \leqslant i \leqslant n}$, $f(x)$ sont des fonctions numériques continues (resp. holomorphes) définies dans un intervalle fermé $I=[a, b]$ de la droite réelle $R$ (resp. dans un domaine connexe $U d u$ plan complexe $C$, limité par un contour $\Gamma$ ).

Soit $G(x, s)$ la solution de l'équation homogène associée (23) qui vérifie pour $x=s \in[a, b]$ les conditions de Cauchy (24), où l'on fait $x=s$ après dérivation. 
Dans ces conditions, la fonction $G(x, s)$ donne non seulement la solution

$$
Z(x)=\int_{x_{0}}^{x} G(x, s) f(s) d s,
$$

d'une part, pour l'équation non homogène (1), avec $Z^{(k)}\left(x_{0}\right)=0 \quad(k=1,2, \ldots$ $\ldots, n-1)$ mais aussi un système fondamental $\left(Y_{i}(x)\right)_{1 \leqslant i \leqslant n}$ de solutions de l'équation homogène associée, défini sous forme explicite par (25) où $L_{s}\left[\left(s-x_{0}\right)^{i-1} /(i-1) !\right]$ est l'opérateur linéaire

$$
\begin{aligned}
L_{s} \cdot \frac{\left(s-x_{0}\right)^{i-1}}{(i-1) !}= & a_{1}(x) \frac{d^{n-1}}{d x^{n-1}}\left(\frac{\left(s-x_{0}\right)^{i-1}}{(i-1) !}\right)+ \\
& +a_{2}(x) \frac{d^{n-2}}{d x^{n-2}}\left(\frac{\left(s-x_{0}\right)^{i-1}}{(i-1) !}\right)+\ldots+a_{n}(x) \frac{\left(s-x_{0}\right)^{i-1}}{(i-1) !} .
\end{aligned}
$$

En outre, le système fondamental $\left(Y_{i}(x)\right)_{1 \leqslant i \leqslant n}$ vérifie les conditions $Y_{i}^{(k-1)}\left(x_{0}\right)=\delta_{i k}$, $\delta_{i k}$ étant le symbole de Kronecker.

On voit done que le problème de la résolution d'une équation différentielle linéaire d'ordre $n$ est ainsi ramené à celui de la détermination de la fonction $G(x, s)$, solution de l'équation homogène (23), qui vérifie les conditions de Cauchy (24) pour un point $s$ arbitrairement choisi dans l'intervalle $[a, b]$. Pour cela, on peut, par exemple, partir de la formule (16), ce qui, compte tenu de $(20)$, mène pour $G(x, s)$ à l'équation intégro-différentielle

$$
G(x, s)=\frac{(x-s)^{n-1}}{(n-1) !}+\int_{s}^{x} \frac{\left(x_{-}-t\right)^{n-1}}{(n-1) !} L_{t} G(t, s) d t
$$

que l'on peut résoudre, en général, par la méthode des approximations successives.

Remarque. On sait que dans le calcul des noyaux itérés qui entrent dans l'expression du noyau résolvant d'une équation intégrale du type de Volterra, on applique la formule de Dirichlet

$$
\int_{a}^{x} d u \int_{a}^{u} f(u, v) d v=\int_{a}^{x} d v \int_{v}^{x} f(u, v) d \dot{u}
$$

$f(u, v)$ étant une fonction numérique continue définie dans un domain compact de $R \times R$.

Pour que les théorèmes 1 et 2 puissent s'appliquer au cas où les fonctions $\left(a_{i}(x)\right)_{1 \leqslant i \leqslant n}$ et $f(x)$ sont holomorphes dans un domaine connexe $\boldsymbol{U}$ du plan complexe $C$, limité par une courbe fermée $\Gamma$, il faut montrer que la formule de Dirichlet $(\alpha)$ est valable aussi dans le cas où $\dot{f}(u, v)$ est une fonction holomorphe des variables $u \epsilon A$ et $v \epsilon B, A$ et $B$ étant deux domaines connexes et fermés du plan complexe $C$.

En effet, soient $a$ et $x$ deux nombres complexes. Considérons les deux intégrales

$$
F_{1}(x)=\int_{a}^{x} d u \int_{a}^{u} f(u, v) d v \quad \text { et } \quad F_{2}(x)=\int_{a}^{x} d v \int_{v}^{x} f(u, v) d u=\int_{a}^{x} \varphi(x, v) d v
$$

où $f(u, v)$ est une fonction holomorphe pour $u \in A$ et $v \epsilon B$.

Nous allons montrer que $F_{1}=F_{2}$ (formule de Dirichlet pour les fonctions holomorphes).

En prenant les dérivées de $F_{1}$ et $F_{2}$ on a

$$
F_{1}^{\prime}(x)=\int_{a}^{x} f(x, v) d v \quad \text { et } \quad F_{2}^{\prime}(x)=\varphi(x, x)+\int_{a}^{x} \varphi_{x}^{\prime}(x, v) d v
$$

mais $\varphi(x, v)=\int_{\nu}^{x} f(u, v) d v$, donc $\varphi(x, x)=0$.

D'autre part on a $\varphi_{x}^{\prime}(x, v)=f(x, v)$, ce qui donne

$$
F_{2}^{\prime}(x)=\int_{a}^{x} f(x, v) d v
$$

Donc $F_{1}^{\prime}(x)=F_{2}^{\prime}(x)$, d'où $F_{1}(x)=F_{2}(x)+C^{t e}$, et comme pour $x=a$, on a $F_{1}(a)=F_{2}(a)=0$, il vient $F_{1}(x)=F_{2}(x)$, c. q. f. d.

Exemple. Appliquons les résultats précédents à l'équation

$$
y^{\prime \prime}=-a^{2} y+f(x) \text {. }
$$

On a $G_{x^{2}}^{\prime \prime}(x, s)=-\alpha^{2} G(x, s)=L_{x} G(x, s)$ d'où, pour $G(x, s)$, compte tenu de (28), l'équation intégrale

$$
G(x, s)=(x-s)-\alpha^{2} \int_{s}^{x}(x-t) G(t, s) d t
$$

dont la solution est $G(x, s)=[\sin \alpha(x-s)] / \alpha$.

Les formules (25) donnent le système fondamental

$$
Y_{1}(x)=1-\alpha \int_{x_{0}}^{x} \sin \alpha(x-t) d t=\cos \alpha\left(x-x_{0}\right)
$$

$$
Y_{2}(x)=x-x_{0}-\alpha \int_{x_{0}}^{x} \sin \alpha(x-t)\left(t-x_{0}\right) d t=\left[\sin \alpha\left(x-x_{0}\right)\right] / \alpha
$$


La solution particulière de l'équation non homogène étant

$$
Z(x)=\frac{1}{\alpha} \int_{x_{0}}^{x} \sin \alpha(x-s) f(s) d s
$$

on trouve pour la solution générale de (29)

$$
y(x)=c_{1} \cos \alpha\left(x-x_{0}\right)+\frac{c_{2}}{\alpha} \sin \alpha\left(x-x_{0}\right)+\frac{1}{\alpha} \int_{x_{0}}^{x} \sin \alpha(x-s) f(s) d s .
$$

\section{Travaux cités}

[1] E. Goursat, Oours d'Analyse Mathématique, Paris 1911. 